\title{
NEW METALLICITY SCALE OF ELLIPTICAL GALAXIES
}

\section{O.K. SIL'CHENKO}

Sternberg Astronomical Institute

University av.13, Moscow 119899, Russia.E-mail: olga@sai.msu.su

This work presents a new calibration of the metallicity scale for elliptical galaxies based on new observational data. I use my observations with the 1000 -channel IPCS of the $6 \mathrm{~m}$ telescope. The spectra for 100 galaxies are published elsewhere (Sil'chenko \& Shapovalova, 1989; Sil'chenko, 1990). A subsample of 30 ellipticals has become a base for the new metallicity scale.

To determine metallicities, I use 3 characteristics of the $M g I \lambda 5175$ absorption line: an equivalent width, a magnesium index $I_{M g}$ (Zasov \& Sil'chenko, 1983), and the already known $M g_{2}$ (Burstein et al., 1984). The metallicity calibration formulae and individual metallicity estimations can be found in my more detailed paper (Sil'chenko, 1994).

My first aim was to look for a metallicity-luminosity relation by using my sample of ellipticals. The recent work of $\mathrm{Da}$ Costa (1992) presents such a relation for dwarf spheroidal galaxies. The metallicity-luminosity relation for my sample of giant-to-intermediate elliptical galaxies perfectly joins that for dwarf spheroidal galaxies. The existence of the united relation for all old-population galaxies with $M_{V}$ from -9 to -23 mag may be evidence for a single formation mechanism for most spheroidal galaxies.

The individual metallicity deviations from the mean relation correlate with $a_{4}$ : boxy galaxies possess metallicity excess. It implies that merging may play a role in the evolution of some galaxies; but obviously it is a merging of gas-rich objects accompanying by a star formation burst.

\section{References}

Burstein, D., et al. (1984) Astrophys. J., 287, pp. 586-609

Da Costa, G.S. (1992) The Stellar Populations of Galaxies. IAU Symp. No.149/Eds.

Barbuy, B. and Renzini, A. Dordrecht: Kluwer Acad. Publ., pp. 191-200

Sil'chenko, O.K., Shapovalova, A.I. (1989) Soobsch. SAO RAS, No. 60, pp. 44-56

Sil'chenko, O.K. (1990) Soobsch. SAO RAS, No. 65, pp. 75-90

Sil'chenko, O.K. (1994) Astron. Zh., 71, pp. 7-16

Zasov, A.V. and Sil'chenko, O.K. (1983) Astron. Zh., 60, pp. 1063-1072 